\title{
Scrutinizing the pion condensed phase
}

\author{
Stefano Carignano ${ }^{1}$, Luca Lepori ${ }^{2,3}$, Andrea Mammarella ${ }^{1}$, Massimo Mannarelli ${ }^{1}$, and Giulia Pagliaroli ${ }^{1,4}$ \\ 1 INFN, Laboratori Nazionali del Gran Sasso, Via G. Acitelli, 22, I-67100 Assergi (AQ), Italy \\ 2 Dipartimento di Fisica e Astronomia, Universitá di Padova, Via Marzolo 8, I-35131 Padova, Italy \\ 3 Dipartimento di Scienze Fisiche e Chimiche, Universitá dell'Aquila, via Vetoio, I-67010 Coppito-L'Aquila, Italy \\ ${ }^{4}$ Gran Sasso Science Institute, Viale Francesco Crispi, 7, L'Aquila, Italy
}

Received: date / Revised version: date

\begin{abstract}
When the isospin chemical potential exceeds the pion mass, charged pions condense in the zeromomentum state forming a superfluid. Chiral perturbation theory provides a very powerful tool for studying this phase. However, the formalism that is usually employed in this context does not clarify various aspects of the condensation mechanism and makes the identification of the soft modes problematic. We re-examine the pion condensed phase using different approaches within the chiral perturbation theory framework. As a first step, we perform a low-density expansion of the chiral Lagrangian valid close to the onset of the Bose-Einstein condensation. We obtain an effective theory that can be mapped to a Gross-Pitaevskii Lagrangian in which, remarkably, all the coefficients depend on the isospin chemical potential. The lowdensity expansion becomes unreliable deep in the pion condensed phase. For this reason, we develop an alternative field expansion deriving a low-energy Lagrangian analog to that of quantum magnets. By integrating out the "radial" fluctuations we obtain a soft Lagrangian in terms of the Nambu-Goldstone bosons arising from the breaking of the pion number symmetry. Finally, we test the robustness of the second-order transition between the normal and the pion condensed phase when next-to-leading-order chiral corrections are included. We determine the range of parameters for turning the second-order phase transition into a first-order one, finding that the currently accepted values of these corrections are unlikely to change the order of the phase transition.
\end{abstract}

PACS. 11.30.Rd Chiral symmetries - 12.39.Fe Chiral Lagrangians - 67.25.D Superfluid phase

\section{Introduction}

Systems with a nonvanishing isospin chemical potential, $\mu_{I}$, are very good playgrounds for gaining insight on quantum chromodynamics (QCD) in the nonperturbative regime. Indeed, several complementary approaches can be employed to study them, possibly leading to a more solid understanding of their properties. The first results on the properties of matter at vanishing temperature as a function of $\mu_{I}$ have been obtained by chiral perturbation theory $(\chi \mathrm{PT})$, see [1,2]. At not too large $\mu_{I}$, lattice QCD simulations of pions (and kaons) are feasible and have been developed in 3, 4, 5, 6, 7, 8, The Nambu-Jona Lasinio (NJL) models can be used in a wide range of the isospin chemical potential [9, 10, 11, 12, 13, 14, 15] and random matrix models have been developed as well [16,17. Perturbative methods have been used in [18 for addressing a region outside the realm of $\chi \mathrm{PT}$, but possibly reachable by future lattice QCD simulations. Finite temperature effects were also considered in several works [15, 19, 20, 21, 22. Non-homogeneous phases at finite isospin densities have been studied in [23, 24, 25, 26]. Clearly, all of these models have a drawback. For example, present lattice sim- ulations at $\mu_{I} \gtrsim 2 m_{\pi}$ have large errors and NJL model results depend on the parameter sets employed.

The isospin chemical potential has several effects on hadronic matter. Not only it induces a Zeeman-like mass splitting within isomultiplets, but it can also rotate the quark-antiquark condensate. The latter phenomenon is called pion condensation, because it is characterized by the breaking of the $U(1)$ global symmetry corresponding to the conservation of the pion number and the occurrence of a Bose-Einstein condensate (BEC) of pions. Since condensed pions are charged, the system is a superfluid of charged particles, that is, an electromagnetic superconductor 27. Using the leading order (LO) $\chi \mathrm{PT}$ Lagrangian it was found that at $\mu_{I}=m_{\pi}$ a second order phase transition between the normal and the pion-condensed $(\pi c)$ phase occurs [1].

In this work we will use a realization of $\chi \mathrm{PT}$ that includes only pions $28,29,30,31$, meaning that we will restrict ourselves to $\left|\mu_{I}\right|<m_{\rho} \sim 770 \mathrm{MeV}$. We also assume that the strange quark chemical potential is so small that it does not allow the appearance of kaons. The present analysis can be easily extended to the kaon condensed phase, see for example the discussion in 32. Since we only 
deal with mesons, the baryonic chemical potential $\mu_{B}$ does not appear in the Lagrangian. We shall implicitly assume that $\mu_{B}$ is below the nucleon mass, see for example the discussion in 2] and in [27.

From the $\chi \mathrm{PT}$ perspective, the possibility of comparing its results with those obtained using different methods would allow for a tuning of its parameters and lead to a better understanding of the ground state and the low-energy properties of the system. As a remarkable example, in the lattice QCD simulations of [5] it was found that the ratio between the energy density and the StefanBoltzmann energy density has a peak at $\mu_{I}^{\text {peak }} \simeq 1.27 m_{\pi}$. In 32] it has been shown that this peak structure can be accurately described using the LO $\chi \mathrm{PT}$ Lagrangian, with an analytic result for the peak position $\mu_{I}^{\text {peak }}=$ $(\sqrt{13}-2)^{1 / 2} m_{\pi} \simeq 1.27 m_{\pi}$. This peak seems to be related with the saturation of the pion condensate. Moreover, we have indications from the LO $\chi \mathrm{PT}$ Lagrangian that the system should make a smooth crossover from the BEC phase to a Bardeen-Cooper-Schrieffer (BCS) phase at $\bar{\mu}_{I} \simeq \sqrt{3} m_{\pi}$, in agreement with the NJL findings of [15], giving $\bar{\mu}_{I} \sim 1.6-2 m_{\pi}$, depending on the parameter set considered.

Although the $\chi \mathrm{PT}$ expansion is extremely powerful for the determination of the ground state properties and of the low-lying excited states, the physical interpretation of the $\pi c$ phase is, in our opinion, not so clear. One of the reasons is that for $\mu_{I}=0$ the charged pions are charge conjugate fields. Turning on the isospin chemical potential explicitly breaks this symmetry, therefore the identification of the physical states is cumbersome. The situation becomes even more complicated in the $\pi c$ phase, in which the Lagrangian takes a non trivial expression and the lowlying states are given by complicated combinations of the pion fields, see for example [27.

In order to shed some light on the $\pi c$ phase, we will provide different expansions of the $\chi \mathrm{PT}$ Lagrangian, aiming at a more accessible physical interpretation of known results obtained at $T=0$. In particular, we will rewrite the Lagrangian in forms similar to those obtained in condensed matter systems or in the study of superfluid phenomena. In the context of weakly interacting bosonic systems, various different approaches can be used, see [33] for a review. Close to the BEC onset we derive a low-density expansion having exactly the form of a Gross-Pitaevskii (GP) equation. This approach is similar to the one developed in standard dilute bosonic systems 33] considering a $\sqrt{n a^{3}}$ expansion, where $n$ is the number density and $a$ is the $s$-wave scattering length. We name it the lowdensity expansion because, as we will see, the tree-level $2 \rightarrow 2$ scattering amplitude tends to a constant nonvanishing value at the BEC phase transition point. Therefore, the actual control parameter in the $\pi \mathrm{c}$ phase is the density of pions in the condensate. Remarkably, both the effective chemical potential, the effective mass and the $2 \rightarrow 2$ scattering amplitude of the GP Lagrangian obtained by the low-density expansion depend on $\mu_{I}$. This identification clarifies one of the reasons why the description of the $\pi c$ phase is complicated: by changing $\mu_{I}$, all of these quan- tities simultaneously change. For this part of the discussion we will restrict ourselves to leading-order results, but next-to-leading order terms in the low-density expansion can be straightforwardly determined.

For larger values of $\mu_{I}$, the low-density expansion breaks down. The basic reason is that the system is no more dilute, with a large number of pions occupying the ground state. In order to gain insight on the properties of the system we provide an alternative description of the $\pi c$ phase, similar to the low-energy description of quantum magnets. We identify two different excitations: the radial, or Higgs, mode corresponding to amplitude fluctuations of the pion condensate, and the phase oscillation of the condensate corresponding to the massless Nambu-Goldstone boson (NGB). This mode is associated with the breaking of the global $U(1)$ symmetry related to the pion number conservation and is also known in condensed matter physics as the Anderson-Bogoliubov mode; it can also be interpreted as the quasiparticle associated with the propagation of pressure perturbations, thus we sometimes call it the phonon. We find a soft Lagrangian similar to the Heisenberg model for quantum magnets in which isospin plays the role of spin in condensed matter. Therefore, the pion condensed phase can be thought as an ordered magnetic phase with isospin aligned along the $\mu_{I}$ direction. By integrating out the radial fluctuations we derive all the interaction terms and the surface terms of the soft Lagrangian. In particular, since the isospin chemical potential explicitly breaks the Lorentz boost invariance but not the rotation invariance, the kinetic term and the interaction terms can be written using an analogue model of gravity.

Finally, we scrutinize the effect of the next-to-leadingorder (NLO) chiral corrections in the static Lagrangian. We examine, for the first time, the effect of these corrections on the phase transition between the normal and the pion condensed phases. After including these corrections, the phase transition happens at values of $\mu_{I}$ of the order of the NLO pion mass. We also determine the range of the NLO low-energy constants necessary for changing the order of the transition between the normal and the $\pi \mathrm{c}$ phases.

The present paper is organized as follows. In Sec. 2 we summarize some of the most interesting properties of the $\pi c$ phase at vanishing temperature obtained in the literature. In Sec. 3 we present a low-energy effective theory valid close to the normal phase-BEC phase transition. In Sec. 4 we provide an alternative description of the $\pi c$ phase valid within the $\chi \mathrm{PT}$ validity range. In Sec. 5 we consider the effect of the NLO corrections in the static Lagrangian. We draw our conclusions in Sec. 6. In the Appendix A we speculate on the existence of self-bound pion stars. In the Appendix B we clarify some aspects of the alternative procedure developed in Sec. 4 . 


\section{Brief summary of the standard $\chi \mathrm{PT}$ results on the pion-condensed phase}

The $\mathcal{O}\left(p^{2}\right) \chi \mathrm{PT}$ Lorentz-invariant Lagrangian describing the interaction of pions with an external vector field, $v_{\mu}$, can be written as follows [2,28, 31

$$
\mathcal{L}_{2}=\frac{f_{\pi}^{2}}{4} \operatorname{Tr}\left(D_{\nu} \Sigma D^{\nu} \Sigma^{\dagger}\right)+\frac{f_{\pi}^{2} m_{\pi}^{2}}{4} \operatorname{Tr}\left(\Sigma+\Sigma^{\dagger}\right)
$$

where

$$
D_{\mu} \Sigma=\partial_{\mu} \Sigma-\frac{i}{2}\left[v_{\mu}, \Sigma\right]
$$

is the covariant derivative and $\Sigma$ is an $S U(2)$ matrix collecting the pion fields. Any explicit expression for $\Sigma$ will correspond to a given parameterization of the pion fields, although results for physical observables are independent of the parameterization of $\Sigma$ used [34, 35, 36]. We will exploit this freedom to develop different expressions of the chiral Lagrangian. In the standard description, see for example [2], the pion degrees of freedom are introduced by considering

$$
\Sigma=u \bar{\Sigma} u \quad \text { with } \quad u=e^{i \boldsymbol{\sigma} \cdot \boldsymbol{\varphi} / 2},
$$

where $\varphi_{i}(i=1,2,3)$ are real scalar fields, $\sigma_{i}$ are the Pauli matrices and

$$
\bar{\Sigma}=e^{i \boldsymbol{\alpha} \cdot \boldsymbol{\sigma}}=\cos \alpha+i \boldsymbol{n} \cdot \boldsymbol{\sigma} \sin \alpha,
$$

is the most general $S U(2)$ vacuum, with $\alpha$ and $\boldsymbol{n}$ variational parameters to be determined by maximizing the static Lagrangian. The low-energy constants (LECs) $f_{\pi}$ and $m_{\pi}$ in Eq. (1) correspond to the pion decay constant and to the pion mass, respectively. These LECs can be related to microscopic quantities of the underlying quark model, see for example [28, 29,30,31. We assume that all three pions have exactly the same mass at vanishing chemical potential. The isospin chemical potential can be introduced as the time component of the external vector field, that is

$$
v_{\mu}=\mu_{I} \delta_{\mu 0} \sigma_{3},
$$

and it is considered as a tunable parameter in the grandcanonical approach. Clearly, it breaks Lorentz-boost invariance and isospin symmetry, because it indicates a privileged reference frame as well as a direction in isospin space.

In the normal phase $\alpha=0$, meaning that $\bar{\Sigma}=I$ and the only effect of introducing $\mu_{I}$ is a Zeeman-like energy splitting proportional to the isospin charge. Thus, the charged pion fields corresponding to

$$
\pi_{ \pm}=\frac{\varphi_{1} \mp i \varphi_{2}}{\sqrt{2}}
$$

have effective masses

$$
m_{\pi_{ \pm}}=m_{\pi} \mp \mu_{I}
$$

and since the neutral pion field $\pi^{0}=\varphi_{3}$ has vanishing isospin, it follows that $m_{\pi^{0}}=m_{\pi}$. In the following we will assume for definiteness $\mu_{I} \geq 0$ and we will use the adimensional quantity

$$
\gamma=\frac{\mu_{I}}{m_{\pi}}
$$

as control parameter to characterize the strength of the isospin chemical potential.

In principle, a system of pions decays into leptons. Therefore, in order to perform a study like ours, one typically neglects electroweak interactions (usually by considering time scales much shorter than their characteristic one). However, let us emphasize that for $\mu_{I}>m_{\pi}-m_{e}$, where $m_{e}$ is the electron mass, the effective $m_{\pi_{+}}$is so small that it cannot decay into leptons [27. The corresponding pion number, $N_{\pi_{+}}$, is thus conserved even when including electroweak interactions. However, at $\gamma=1$ the $\pi_{+}$ becomes massless and the $U(1)$ symmetry corresponding to $N_{\pi_{+}}$conservation is spontaneously broken: the system becomes a superfluid. Since the condensed bosons are electrically charged, the resulting phase is actually a superconductor [27. This symmetry breaking mechanism can be described by maximizing the $\mathcal{O}\left(p^{2}\right)$ ground state Lagrangian. For $\gamma>1$, the energetically favored phase is characterized by $\cos \alpha=1 / \gamma^{2}$, meaning that the large isospin chemical potential has changed the property of the vacuum.

The identification of the ground state having $\alpha \neq 0$ with a superfluid is based on several facts that we briefly review. It is possible to show that the chiral condensate is rotated to a pion condensate, more specifically 2

$$
\begin{array}{r}
\langle\bar{u} u\rangle=\langle\bar{d} d\rangle \propto \cos \alpha, \\
\left\langle\bar{d} \gamma_{5} u+\text { h.c. }\right\rangle \propto \sin \alpha,
\end{array}
$$

where $u$ and $d$ are up and down quarks respectively (color and spinorial indices have been suppressed). A nonvanishing pion condensate implies that the vacuum does not annihilate the isospin charge. The condensed pions contribute to the total pressure and density of the system. Since we are considering vanishing temperatures, these contributions are due to the occupation of the zero energy state by a macroscopic number of particles. The normalized pressure (obtained subtracting the vacuum pressure) and the number density are respectively given by [1, 2

$$
P=\frac{f_{\pi}^{2} m_{\pi}^{2}}{2} \gamma^{2}\left(1-\frac{1}{\gamma^{2}}\right)^{2}, \quad n_{I}=f_{\pi}^{2} m_{\pi} \gamma\left(1-\frac{1}{\gamma^{4}}\right)
$$

leading to the $\mathcal{O}\left(p^{2}\right)$ equation of state 32

$$
\epsilon(P)=-P+2 \sqrt{P\left(2 f_{\pi}^{2} m_{\pi}^{2}+P\right)} .
$$

The isospin number density, which is equivalent to the electric charge one, exactly corresponds to the number density of particles in the ground state.

Regarding the excitations, it can be shown that there exists a flat direction of the potential, which is a typical feature of the BEC phase because it is associated with the existence of NGBs. Indeed, by a variational procedure it 
is possible to show that the unit vector $\boldsymbol{n}$ in Eq. (4) has to be orthogonal to the direction taken by the vector field $v_{\mu}$ in isospin space 27. This residual $O(2)$ isospin symmetry corresponds to the flat direction of the potential. The low-energy excitations are given by two orthogonal combinations of the pion fields; the corresponding dispersion laws can be found in 27. The dispersion law of the NGB mode is given by

$$
E=\frac{m_{\pi}}{\sqrt{2} \gamma} \sqrt{3+\gamma^{4}+2 p^{2} \frac{\gamma^{2}}{m_{\pi}^{2}}-\sqrt{\left(3+\gamma^{4}\right)^{2}+16 p^{2} \frac{\gamma^{2}}{m_{\pi}^{2}}}}
$$

which can be expanded in two different regimes:

$$
\begin{array}{ll}
E=\frac{p^{2}}{2 m_{\pi}} & \text { for } \gamma=1, \\
E=c_{s} p+\mathcal{O}(p)^{3} & \text { for } \gamma>1,
\end{array}
$$

where

$$
c_{s}=\sqrt{\frac{\gamma^{4}-1}{\gamma^{4}+3}}
$$

is the sound speed. The above results indicate that this is indeed a gapless mode. However, at the phase transition this mode seems to interpolate between two different excitations. Indeed, for $\gamma=1$ it has a quadratic dispersion law and thus describes the $\pi_{+}$that becomes massless at the phase transition point. For $\gamma>1$, it describes a mode propagating with the speed of sound (which can as well be obtained from the equation of state, Eq. (12) ) and should therefore correspond to the phonon. Technically, this interpolation is possible because the sound speed vanishes at the phase transition point.

Although the above discussion is formally correct, it does not actually illustrate the symmetry breaking mechanism in detail. As we will see, the procedure for obtaining the ground state includes the nonperturbative interactions of the $\mathcal{O}\left(p^{2}\right)$ Lagrangian and this is one of the reasons why the result looks cumbersome. Moreover, the soft Lagrangian for the NGB is difficult to identify, because the massless mode is a combination of the two charged ones. Finally, in the standard description of the broken phase there is still a massive mode, which should be integrated out in order to obtain the soft Lagrangian. In the following section we will discuss the symmetry breaking mechanism using a low-energy expansion, valid in the normal phase and close to the BEC phase transition, which has a more intuitive interpretation. In Sec. 4 we present a method for obtaining the soft Lagrangian for any $\gamma$ within the $\chi \mathrm{PT}$ range.

\section{Low energy description of the normal phase-BEC phase transition}

The $U(1)$ symmetry breaking corresponding to the violation of the pion number conservation can be described by considering the standard expression of the chiral fields

$$
\Sigma=e^{i \sigma \cdot \varphi},
$$

but assuming a nonzero vacuum expectation value (vev) of one of the charged fields. Since the $\pi_{0}$ will not play any role, we restrict our analysis to the charged fields, meaning that we will only consider the $\varphi_{1}$ and $\varphi_{2}$ components. By expanding the $\mathcal{O}\left(p^{2}\right)$ Lagrangian in Eq. (1) including terms up to $\varphi^{4}$, we find

$$
\begin{aligned}
\mathcal{L} & =f_{\pi}^{2} m_{\pi}^{2}+i f_{\pi}^{2} \mu_{I}\left(\pi_{-} \partial_{0} \pi_{+}-\pi_{+} \partial_{0} \pi_{-}\right)+f_{\pi}^{2}\left(\partial_{\nu} \pi_{+} \partial^{\nu} \pi_{-}\right) \\
& -f_{\pi}^{2}\left(m_{\pi}^{2}-\mu_{I}^{2}\right) \pi_{+} \pi_{-}+\frac{f_{\pi}^{2}}{6}\left(m_{\pi}^{2}-4 \mu_{I}^{2}\right)\left(\pi_{+} \pi_{-}\right)^{2}+\ldots
\end{aligned}
$$

where the pion fields have the same expression reported in Eq. (6) and we have neglected derivative terms $\mathcal{O}\left(\varphi^{3}\right)$ and higher. The presence of terms coupling $\pi_{+}$and $\pi_{-}$fields makes the study of the Lagrangian complicated. However, close to the phase transition it is possible to consider excitations at arbitrarily small energies because a massless mode exists, see also Eq. (32) below. Therefore, in this region, we can restrict the analysis to the terms with the lowest derivative power, leading to a further simplified soft-pion Lagrangian

$$
\begin{aligned}
\mathcal{L} & =f_{\pi}^{2} m_{\pi}^{2}+i f_{\pi}^{2} \mu_{I}\left(\pi_{-} \partial_{0} \pi_{+}-\pi_{+} \partial_{0} \pi_{-}\right)-f_{\pi}^{2}\left(\nabla \pi_{+} \cdot \nabla \pi_{-}\right) \\
& -f_{\pi}^{2}\left(m_{\pi}^{2}-\mu_{I}^{2}\right) \pi_{+} \pi_{-}+\frac{f_{\pi}^{2}}{6}\left(m_{\pi}^{2}-4 \mu_{I}^{2}\right)\left(\pi_{+} \pi_{-}\right)^{2} .
\end{aligned}
$$

From this, one obtains the following equations of motion for the $\pi_{ \pm}$fields:

$$
\begin{aligned}
\pm 2 i f_{\pi}^{2} \mu_{I} \partial_{0} \pi_{\mp} & =-f_{\pi}^{2} \nabla^{2} \pi_{\mp}+f_{\pi}^{2}\left(m_{\pi}^{2}-\mu_{I}^{2}\right) \pi_{\mp} \\
& -\frac{1}{3} f_{\pi}^{2}\left(m_{\pi}^{2}-4 \mu_{I}^{2}\right)\left(\pi_{+} \pi_{-}\right) \pi_{\mp},
\end{aligned}
$$

showing that $\pi_{+}$corresponds to the low-energy particle state and $\pi_{-}$to the low-energy antiparticle one. As already noted in [37, there is only one independent degree of freedom, because in the low-energy limit $\pi_{+}$and $\pi_{-}$are conjugate fields. For any $\mu_{I}>0$ we can define

$$
\psi=\sqrt{2 f_{\pi}^{2} \mu_{I}} \pi_{+},
$$

thus Eq. (19) takes the form of a Gross-Pitaevskii (GP) Lagrangian

$\mathcal{L}_{\mathrm{GP}}=f_{\pi}^{2} m_{\pi}^{2}+i \psi^{*} \partial_{0} \psi+\mu_{\mathrm{eff}} \psi^{*} \psi-\frac{g}{2}\left|\psi^{*} \psi\right|^{2}+\psi^{*} \frac{\nabla^{2}}{2 M} \psi$,

with

$$
\mu_{\mathrm{eff}}=\frac{\mu_{I}^{2}-m_{\pi}^{2}}{2 \mu_{I}}, \quad g=\frac{4 \mu_{I}^{2}-m_{\pi}^{2}}{12 f_{\pi}^{2} \mu_{I}^{2}}, \quad M=\mu_{I},
$$

the relevant GP coefficients. It is quite striking to see the very nontrivial effect of the isospin chemical potential. Not only it changes the effective chemical potential, but it also changes the boson-boson coupling and the coefficient of the Laplacian operator. The effective coupling constant is related to the $\pi^{+} \pi^{+}$scattering length, $a$, by the standard GP relation $a \propto g m_{\pi}$. However, the scattering amplitude of different pions cannot be simply determined in this way 
because isospin is explicitly broken in the Lagrangian, see Eqs. (11) and (5). In particular, no simple relation exists between the scattering amplitudes in the various isospin channels.

Since the mode is bosonic, the effective chemical potential in the unbroken phase must be nonpositive, and indeed this happens for $\mu_{I} \leq m_{\pi}$. Since we are working at $T=0$, the region $\mu_{I}<m_{\pi}$ corresponds to the vacuum with no pions, whereas for $\mu_{I}=m_{\pi}$ pions appear and the effective chemical potential vanishes. Whether this condition corresponds to the onset of the BEC regime depends on the sign of the effective interaction. For attractive interactions the system should collapse, while for repulsive ones it becomes superfluid. From our analysis, Eq. (23) indicates that the interaction coupling becomes positive for $\mu_{I}>m_{\pi} / 2$, thus the interaction between the $\psi$ fields is repulsive and the system is expected to turn into a BEC when the effective chemical potential vanishes. It is quite remarkable that well before reaching the transition point, the interaction turns from attractive to repulsive. It might be of interest to study within this GP model what happens at a small nonvanishing temperature for $\mu_{I}<m_{\pi} / 2$, corresponding to a system in which few thermal excitations interact with a negative scattering length. In this case the GP Lagrangian in Eq. (22) is still valid because the neglected derivative interactions are thermally suppressed. This approximation is expected to break down only close to $\mu_{I}=m_{\pi} / 2$, when $g$ vanishes. We postpone this study to future work.

From Eq. (22), we have the potential

$$
V(n)=\mu_{\mathrm{eff}} n-\frac{g}{2} n^{2},
$$

where $n=\psi^{*} \psi$. The ground state number density is obtained from $\partial V / \partial n=0$, leading to

$$
n=6 f_{\pi}^{2} m_{\pi} \gamma \frac{\gamma^{2}-1}{4 \gamma^{2}-1}
$$

where $\gamma$ has been defined in Eq. (8). Close to the BEC phase transition we can write

$$
\gamma=1+\varepsilon
$$

with $\varepsilon \ll 1$, and we obtain the approximate expression

$$
n=4 \varepsilon f_{\pi}^{2} m_{\pi} .
$$

The adimensional parameter $\varepsilon$ in Eq. (26) corresponds in cold atoms experiments to the quantity $x=n a^{3}$, with $a$ being the s-wave scattering length of the bosonic atoms or molecules involved in the condensate [38. In particular, the condition $x \ll 1$ assures the validity of the GP description. Since the neglected terms in Eq. (18) are of order $\left(\psi^{*} \psi\right)^{3} \propto \epsilon^{3}$, it follows that the obtained approximation is only valid at the leading order in $\epsilon$. Indeed, the ground state number density in the broken phase is given by Eq. (11), which agrees with Eq. (27) at the leading order in $\varepsilon$. This expansion is therefore a low-density expansion, and is expected to breakdown for large $n$, or more precisely, for $\varepsilon$ of order unity. Physically, the low-density expansion makes sense because close to the $\pi \mathrm{c}$ phase transition the number density of particles is arbitrarily small, thus interactions involving higher order terms are suppressed.

Close to the phase transition point, the normalized pressure is given by

$$
P=\frac{g}{2} n^{2}=2 \varepsilon^{2} f_{\pi}^{2} m_{\pi}^{2},
$$

which agrees with the result reported in Eq. (11) at the leading order in $\varepsilon$. We thus conclude that close to the normal phase-BEC phase transition, the system can be approximated by the low-density expansion of the chiral Lagrangian that can be mapped to the GP Lagrangian of Eq. (22) having

$$
\mu_{\mathrm{eff}}=\varepsilon, \quad g=\frac{1}{4 f_{\pi}^{2}}\left(1+\frac{3}{2} \varepsilon\right), \quad M=m_{\pi}(1+\varepsilon),
$$

and the resulting pressure and ground state number density are respectively

$$
P=2 \varepsilon^{2} f_{\pi}^{2} m_{\pi}^{2}, \quad n=4 \varepsilon f_{\pi}^{2} m_{\pi} .
$$

Regarding the excitations, the equation of motion takes the form of a GP equation

$$
i \partial_{0} \psi=-\frac{\nabla^{2}}{2 \mu_{I}} \psi-\mu_{\mathrm{eff}} \psi+g|\psi|^{2} \psi
$$

thus, neglecting interactions, the dispersion law of the $\psi$ mode is given by

$$
\epsilon(p)=\frac{p^{2}}{2 \mu_{I}}-\mu_{\mathrm{eff}},
$$

which already includes the effect of the effective chemical potential. Thus, the mode is gapless with a quadratic dispersion law at $\gamma=1$, matching the result obtained in the previous section, see Eq. (14), if one expands for small $\epsilon$. Within the GP framework it is also possible to obtain and to better understand the linear dispersion mode given in Eq. (15). Since in the broken phase the interaction is repulsive, the low-energy excitation is a Bogolyubov mode, or NGB, with dispersion law

$$
\epsilon_{\mathrm{NGB}}(p)=p \sqrt{\frac{n g}{\mu_{I}}}=p \sqrt{\varepsilon},
$$

which agrees with the result reported in Eq. (16) considering the expansion in Eq. (26).

The two different limits of the dispersion law reported in Eqs. (14) and (15), do actually correspond to different modes: the first one is the quasiparticle mode becoming massless at the phase transition point, while the second one is the long-wavelength fluctuations corresponding to longitudinal compression mode, which exists because the boson-boson interaction is repulsive.

The second-order phase transition can therefore be described by the standard GP Lagrangian for sufficiently 
small values of $\varepsilon$. It is maybe of interest the fact that at the leading order the effective coupling depends only on $1 / f_{\pi}^{2}$. Moreover, the strength of the interaction increases with $\mu_{I}$. This is the expected behavior for a system evolving towards a BCS phase. Unfortunately, the BEC-BCS crossover is expected to happen at $\gamma \simeq \sqrt{3}$, see 32 , and it is therefore outside the range of the low-density expansion.

\section{Alternative description of the pion condensed phase}

In the previous section we have clarified that the pion condensation mechanism can be better understood by a mapping of the chiral Lagrangian into a GP Lagrangian, which however should include an infinite number of terms for $\mu_{I}$ much larger than $m_{\pi}$. Therefore, we should find an alternative way for treating the system in that regime. In this section we present an approach to the pion condensed phase aiming at a soft Lagrangian density valid for momenta below $\mu_{I}$. We expand our Lagrangian close to the potential minimum to identify the flat direction of the potential. The important aspect is that in the broken phase, where $\gamma>1$, there still exists a massive degree of freedom corresponding to the radial fluctuation of the condensate, which is the so-called Higgs mode. In order to determine the correct expression of the soft Lagrangian we have to integrate out this mode. Clearly, one has first to identify it.

Let us use the following field definition

$$
\Sigma=e^{i \boldsymbol{\sigma} \cdot \boldsymbol{\varphi}}=\cos \rho+i \boldsymbol{\sigma} \cdot \hat{\boldsymbol{\varphi}} \sin \rho,
$$

where $\varphi=\rho \hat{\varphi}$, thus $\rho$ describes the radial field, and $\hat{\varphi}$ is a unit vector field, such that $\hat{\varphi} \cdot \hat{\varphi}=1$. This field decomposition can be thought as obtained from Eq. (4) by promoting $\alpha$ and $\boldsymbol{n}$ to dynamical fields. In the following we will neglect the $\hat{\varphi}_{3}$ field, corresponding to the $\pi_{0}$ direction. We will comment on the $\pi_{0}$ field in the Appendix B. Since we are restricting our analysis to the charged fields, $\boldsymbol{n}$ is a unit vector in a 2 dimensional space and the field $\hat{\varphi}$ corresponds to only one independent degree of freedom describing the fluctuations of this unit vector. For later convenience we define

$$
\hat{\varphi}_{1}=\sqrt{1-\hat{\varphi}_{2}^{2}},
$$

meaning that we will treat $\hat{\varphi}_{2}$ as the independent degree of freedom. As we will see, this fluctuation can be identified with the NGB of the broken phase. We notice that the present procedure resembles closely the one exploited to derive low-energy effective models of $(1+1) d$ quantum magnets, such as the Heisenberg-type ones 39,40, 41, 42 43 . The reason behind this analogy is that although pions are pseudoscalar particles, they have isospin $I=1$. In particular, the charged pions have $I_{3}= \pm 1$ and are therefore analogues to magnets in isospin space. Thus, the system can be thought as an isospin quantum magnet and the broken phase corresponds to an ordered magnetic phase, with quantum isospins aligned along one particular direction in isospin space.

Upon substituting Eq. (34) in Eq. (1), we obtain the $\mathcal{O}\left(p^{2}\right)$ Lagrangian

$$
\begin{gathered}
\mathcal{L}=\frac{f_{\pi}^{2}}{2}\left(\partial^{\mu} \rho \partial_{\mu} \rho+\sin ^{2} \rho \partial^{\mu} \hat{\varphi}_{i} \partial_{\mu} \hat{\varphi}_{i}\right. \\
\left.-2 m_{\pi} \gamma \sin ^{2} \rho \epsilon_{3 i k} \hat{\varphi}_{i} \partial_{0} \hat{\varphi}_{k}\right)-V(\rho),
\end{gathered}
$$

where

$$
V(\rho)=-f_{\pi}^{2} m_{\pi}^{2}\left(\cos \rho+\frac{\gamma^{2}}{2} \sin ^{2} \rho\right),
$$

is the potential. Remarkably, this expression of the $\mathcal{O}\left(p^{2}\right)$ Lagrangian is not obtained by making any expansion in the fields. The potential does not depend on the field $\hat{\varphi}$, showing that, at least at this order, the field $\hat{\varphi}$ does not have non-derivative couplings, as appropriate for NGBs. Clearly, this result is due to the residual $O(2)$ symmetry corresponding to the flat direction of the potential.

The stationary point of the potential can be obtained by solving

$$
\left.\frac{\partial V}{\partial \rho}\right|_{\bar{\rho}}=0 \quad \rightarrow \quad \bar{\rho}=\arccos \frac{1}{\gamma^{2}},
$$

with $\bar{\rho}$ corresponding to a minimum of $V$ for $\gamma>1$. Upon substituting this expression in Eq. (37) one obtains the same expression of the pressure reported in Eq. (11). On the other hand, for $\gamma<1$ the minimum of the potential is in $\rho=0$. Therefore, the $\rho$ field has the typical behavior of the radial mode, which acquires a nontrivial vev in the broken phase. The field $\hat{\varphi}_{2}$ corresponds to a massless fluctuation, that is to the NGB, only in the broken phase when $\sin \bar{\rho} \neq 0$; see the Appendix B. To further clarify how the massive and massless modes appear we expand the Lagrangian in Eq. (36) close to the stationary point.

To tackle the properties of the NGB boson, we first neglect the radial fluctuations. In the broken phase the Lagrangian for the $\hat{\varphi}$ field turns out to be

$$
\mathcal{L}_{\hat{\varphi}}=\frac{f_{\pi}^{2} \sin ^{2} \bar{\rho}}{2}\left(\partial^{\mu} \hat{\varphi}_{i} \partial_{\mu} \hat{\varphi}_{i}-2 m_{\pi} \gamma \epsilon_{3 i k} \hat{\varphi}_{i} \partial_{0} \hat{\varphi}_{k}\right),
$$

and to make contact with the standard expression of the NGB Lagrangian we can make a further variable change,

$$
\theta=\arctan \left(\frac{\hat{\varphi}_{2}}{\hat{\varphi}_{1}}\right)=\hat{\varphi}_{2}+\frac{2 \hat{\varphi}_{2}^{3}}{3}+\mathcal{O}\left(\hat{\varphi}_{2}^{5}\right)
$$

where in the last equality we have used Eq. (35) and conveniently assumed that the condensate is oriented along the 1-direction in isospin space, thus $\hat{\varphi}_{2}$ is a small fluctuation. The soft Lagrangian now reads

$$
\mathcal{L}=\frac{f_{\pi}^{2} \sin ^{2} \bar{\rho}}{2} \partial^{\mu} \theta \partial_{\mu} \theta,
$$

where we have neglected a total derivative. The field $\theta$ is the genuine NGB, or phonon, or Anderson-Bogoliubov 
mode, because it is the phase associated with the rotation of the condensate. Note that the propagation velocity of this mode is not equal to the sound speed $c_{s}$ reported in Eq. (16), but it is equal to 1 . This result depends on the fact that we have completely neglected the interaction of the phonon with the background, or more precisely, with the radial fluctuations.

For considering the radial fluctuation in the broken phase we define $\rho=\bar{\rho}+\chi$, finding that, for $\gamma \geq 1$,

$$
V(\chi)=\frac{f_{\pi}^{2} m_{\pi}^{2}}{\gamma^{2}}\left(-\frac{1+\gamma^{4}}{2}+\frac{\gamma^{4}-1}{2} \chi^{2}\right)+\mathcal{O}\left(\chi^{3}\right),
$$

which shows that the Higgs mode has a nonnegative mass that vanishes at the phase transition point, as appropriate for second order phase transitions.

The Lagrangian that includes both the quadratic fluctuations can be obtained from Eqs. (36) and (42), leading to

$$
\mathcal{L}=-\frac{1}{2} \chi D^{-1} \chi-J \chi+\frac{f_{\pi}^{2} \sin ^{2} \bar{\rho}}{2} \partial_{\mu} \hat{\boldsymbol{\varphi}} \partial^{\mu} \hat{\boldsymbol{\varphi}}+\mathcal{L}_{\mathrm{s}},
$$

where

$$
\begin{aligned}
D^{-1} & =f_{\pi}^{2}\left(\square+m_{\pi}^{2} \frac{\gamma^{4}-1}{\gamma^{2}}\right), \\
J & =f_{\pi}^{2} \mu_{I} \sin (2 \bar{\rho}) \epsilon_{3 i j} \hat{\varphi}_{i} \partial_{0} \hat{\varphi}_{j},
\end{aligned}
$$

and

$$
\mathcal{L}_{\mathrm{s}}=-f_{\pi}^{2} \mu_{I} \sin ^{2}(\bar{\rho})\left(\hat{\varphi}_{1} \partial_{0} \hat{\varphi}_{2}-\hat{\varphi}_{2} \partial_{0} \hat{\varphi}_{1}\right)
$$

corresponds to a surface term. Indeed, using Eq. (35) this term can be written as a total derivative

$$
\mathcal{L}_{\mathrm{S}}=-f_{\pi}^{2} \mu_{I} \sin ^{2} \bar{\rho} \partial_{0} \arcsin \left(\hat{\varphi}_{2}\right) .
$$

Integrating out the radial fluctuations we obtain the $\mathcal{O}\left(p^{2}\right)$ effective Lagrangian describing the propagation and the interaction terms

$$
\begin{aligned}
\mathcal{L}_{\text {eff }} & =f_{\pi}^{2} \sin ^{2} \bar{\rho}\left(\frac{1}{2} \partial_{\mu} \hat{\varphi}_{i} \partial^{\mu} \hat{\varphi}_{i}\right. \\
& \left.+\frac{2}{\gamma^{4}-1}\left(\left(\hat{\varphi}_{1} \partial_{0} \hat{\varphi}_{2}-\hat{\varphi}_{2} \partial_{0} \hat{\varphi}_{1}\right)^{2}\right)\right),
\end{aligned}
$$

which we can expand using Eq. (35) to obtain

$$
\mathcal{L}_{\text {eff }}=\frac{f_{\pi}^{2}}{2} \frac{\gamma^{4}+3}{\gamma^{4}} \sum_{n=0}^{\infty} \hat{\varphi}_{2}^{2 n}\left(\left(\partial_{0} \hat{\varphi}_{2}\right)^{2}-c_{s}^{2}\left(\nabla \hat{\varphi}_{2}\right)^{2}\right),
$$

with the speed of sound given by the same expression reported in Eq (16). It is possible to write the effective Lagrangian in the slightly more compact and suggestive way

$$
\mathcal{L}_{\text {eff }}=\frac{f_{\pi}^{2}}{2} \frac{\gamma^{4}+3}{\gamma^{4}} \sum_{n=0}^{\infty} \hat{\varphi}_{2}^{2 n} g^{\mu \nu} \partial_{\mu} \hat{\varphi}_{2} \partial_{\nu} \hat{\varphi}_{2}
$$

where $g^{\mu \nu}=\operatorname{diag}\left(1,-c_{s}^{2},-c_{s}^{2},-c_{s}^{2}\right)$ is the so-called acoustic metric, see for example the discussion in 44 and the review [45. A remarkable aspect is that the above Lagrangian not only includes the kinetic term but also all the $\mathcal{O}\left(p^{2}\right)$ interaction terms. It is strictly valid for momenta much below $\mu_{I}$, see the Appendix B, and the appearance of the acoustic metric in Eq. (50) is due to the fact that the $\hat{\varphi}_{2}$ field is the analogous of a sound mode. field

Using Eq. (40), we obtain the Lagrangian for the $\theta$

$$
\mathcal{L}=\frac{f_{\pi}^{2}}{2} \frac{\gamma^{4}+3}{\gamma^{4}} g^{\mu \nu} \partial_{\mu} \theta \partial_{\nu} \theta\left(1-3 \theta^{2}+\mathcal{O}\left(\theta^{4}\right)\right),
$$

showing that now this field has the correct propagation velocity. Higher order terms can be obtained by considering higher order terms in Eq. (40).

The proposed procedure, relying on the integration of the radial fluctuations, allows us to easily evaluate the kinetic term and the interaction terms of the $\hat{\varphi}_{2}$ mode, with the additional benefit of having a Lorentz covariant Lagrangian with the effective metric $g^{\mu \nu}$. In principle, in the broken phase it is possible to diagonalize the Lagrangian, as done for example in [2], but this procedure is unnecessarily complicated if one is only interested in momenta below $\mu_{I}$.

\section{NLO corrections}

We now explore a different issue. An important result obtained using the LO chiral Lagrangian is that there is a second order phase transition between the normal phase and the $\pi \mathrm{c}$ phase. This means, among other things, that the chiral condensate is continuously rotated into the pion one, see Eqs. (9) and (10). However, the fact that the rotation is continuous does not seem to rely on any physical reason, meaning that an abrupt tilting in some direction in isospin space by an increasing $\mu_{I}$ would have been a plausible a priori possibility. Consider, for example, that the phase transition between the kaon condensed and the pion condensed phases triggered by an increasing $\mu_{I}$ is of the first order 2 .

To study the robustness of the second-order transition we include NLO $\chi \mathrm{PT}$ corrections. Following [31, for the three-flavor case they are given by

$$
\begin{aligned}
\mathcal{L}_{4} & =L_{1}\left\{\operatorname{Tr}\left[D_{\mu} \Sigma\left(D^{\mu} \Sigma\right)^{\dagger}\right]\right\}^{2} \\
& +L_{2} \operatorname{Tr}\left[D_{\mu} \Sigma\left(D_{\nu} \Sigma\right)^{\dagger}\right] \operatorname{Tr}\left[D^{\mu} \Sigma\left(D^{\nu} \Sigma\right)^{\dagger}\right] \\
& +L_{3} \operatorname{Tr}\left[D_{\mu} \Sigma\left(D^{\mu} \Sigma\right)^{\dagger} D_{\nu} \Sigma\left(D^{\nu} \Sigma\right)^{\dagger}\right] \\
& +L_{4} \operatorname{Tr}\left[D_{\mu} \Sigma\left(D^{\mu} \Sigma\right)^{\dagger}\right] \operatorname{Tr}\left(\chi \Sigma^{\dagger}+\chi^{\dagger} \Sigma\right) \\
& +L_{5} \operatorname{Tr}\left[D_{\mu} \Sigma\left(D^{\mu} \Sigma\right)^{\dagger}\left(\chi \Sigma^{\dagger}+\chi^{\dagger} \Sigma\right)\right] \\
& +L_{6}\left[\operatorname{Tr}\left(\chi \Sigma^{\dagger}+\chi^{\dagger} \Sigma\right)\right]^{2}+L_{7}\left[\operatorname{Tr}\left(\chi \Sigma^{\dagger}-\chi^{\dagger} \Sigma\right)\right]^{2} \\
& +L_{8} \operatorname{Tr}\left(\Sigma \chi^{\dagger} \Sigma \chi^{\dagger}+\chi \Sigma^{\dagger} \chi \Sigma^{\dagger}\right)+H_{2} \operatorname{Tr}\left(\chi \chi^{\dagger}\right)
\end{aligned}
$$

where $L_{i}$, with $i=1, \ldots, 8$, and $H_{2}$ are the relevant LECs encoding properties of the underlying quark theory. In principle one can use these NLO corrections for studying the robustness of any phase transition of the $S U(3)$ 
phase diagram of [4. Nevertheless, in the present paper we focus on the phase transition between the normal phase and the $\pi_{c}$ condensed phase. In this case $\chi=m_{\pi}^{2} \mathbf{1}_{2 \times 2}$ and $\Sigma$ is an $S U(2)$ matrix. Within this restriction, our $\mathcal{L}_{4}$ reduces to the $S U(2)$ expression originally derived in [28, and the $S U(3)$ LECs can be easily mapped to the $S U(2)$ LECs (see for example [46]).

Upon substituting Eq. (4) in $\mathcal{L}_{4}$ we obtain the following expression for the static NLO Lagrangian

$$
\begin{aligned}
\overline{\mathcal{L}}_{\text {stat }}^{\mathrm{NLO}} & =f_{\pi}^{2} m_{\pi}^{2}\left(\cos \alpha+\frac{\gamma^{2}}{2} \sin ^{2} \alpha+2 a \gamma^{4} \sin ^{4} \alpha\right. \\
& \left.+4 b \gamma^{2} \sin ^{2} \alpha \cos \alpha+8 c \cos ^{2} \alpha+2 d\right),
\end{aligned}
$$

where

$$
\begin{array}{ll}
a=\frac{m_{\pi}^{2}}{f_{\pi}^{2}}\left(2 L_{1}+2 L_{2}+L_{3}\right), & b=\frac{m_{\pi}^{2}}{f_{\pi}^{2}}\left(2 L_{4}+L_{5}\right), \\
c=\frac{m_{\pi}^{2}}{f_{\pi}^{2}}\left(2 L_{6}+L_{8}\right), & d=\frac{m_{\pi}^{2}}{f_{\pi}^{2}}\left(H_{2}-2 L_{8}\right),
\end{array}
$$

are the relevant combinations of LECs and where $\gamma$ is given in Eq. (8). Then, by taking $\alpha=0$ we obtain

$$
P_{0}=f_{\pi}^{2} m_{\pi}^{2}(1+8 c+2 d),
$$

the NLO expression of the pressure in the normal phase. At the same order we obtain in the normal phase

$$
\begin{aligned}
m_{\pi, 4}^{2} & =m_{\pi}^{2}(1+16 c-8 b), \\
f_{\pi, 4}^{2} & =f_{\pi}^{2}(1+8 b),
\end{aligned}
$$

corresponding to the standard $\mathcal{O}\left(p^{4}\right)$ values of the pion mass and of the pion decay constant, respectively, see for example [46]. We remark that $P_{0} \neq m_{\pi, 4}^{2} f_{\pi, 4}^{2}$, meaning that the pressure renormalization must be carefully taken into account. If one restricts the analysis to the normal phase, this renormalization is immaterial, because we can subtract an arbitrary constant. However, it is important to take into account the nontrivial renormalization of the pressure $P=P_{\pi c}-P_{0}$, obtained by subtracting the vacuum pressure to the one of the $\pi c$ phase. In particular, the NLO corrections to the pressure of the broken phase depend on $a, b$ and $c$ in a nontrivial way and they can change the value of $\gamma$ for which the transition to the BEC phase takes place. We refer to $\gamma_{c}$ as the value of the $\gamma$ parameter at the phase transition point.

To make the discussion as simple as possible, it is convenient to subtract the normal phase pressure from Eq. (53), obtaining the normalized NLO static Lagrangian

$$
\begin{aligned}
\mathcal{L}_{\text {stat }}^{\mathrm{NLO}} & =f_{\pi}^{2} m_{\pi}^{2}(1-z)\left(\frac{\gamma^{2}}{2}(1+z)+2 a \gamma^{4}\left(1-z^{2}\right)(1+z)\right. \\
& \left.-1+4 b \gamma^{2} z(1+z)-8 c(1+z)\right)
\end{aligned}
$$

where $z=\cos \alpha$. The stationary condition for the Lagrangian is obtained by solving the cubic equation

$$
\gamma^{2} z-1+8 a \gamma^{4}\left(1-z^{2}\right) z+4 b \gamma^{2}\left(3 z^{2}-1\right)-16 c z=0,
$$

considering the appropriate value of $\gamma$. The first order phase transition is obtained when two roots are equal and satisfy $0 \leq z \leq 1$, corresponding to

$$
8 a \gamma_{c}^{4}-12 b \gamma_{c}^{2}+8 c>\frac{\gamma_{c}^{2}}{2}
$$

Before discussing the phase transition in detail, let us notice that for a proper description of the critical value of the isospin chemical potential, one should consider the NLO corrections to the pion mass as well, meaning that the order parameter should be rescaled as follows

$$
\gamma \rightarrow \gamma^{R} \equiv \gamma \frac{m_{\pi}}{m_{\pi, 4}}
$$

with the $\mathcal{O}\left(p^{4}\right)$ rescaled pion mass defined in Eq. (56).

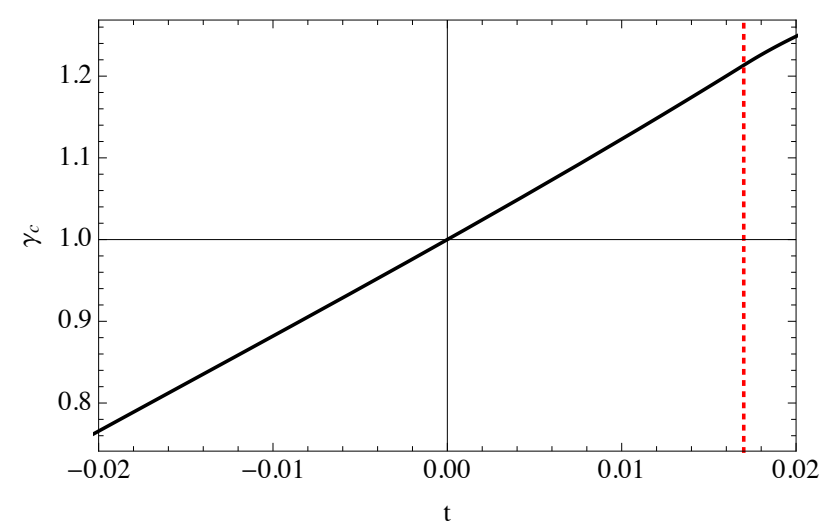

Fig. 1. Effect of the NLO $\chi \mathrm{PT}$ terms on the critical value for the phase transition between the normal phase and the $\pi \mathrm{c}$ phase. The NLO effects are parametrized by $t=a=-b=c$, where $a, b$ and $c$ are the combinations of LECs given in Eq. (54). The solid black line corresponds to the critical value of $\gamma=$ $\mu_{I} / m_{\pi}$. The dotted vertical red line indicates the onset of the first order phase transition.

We note at this point that in spite of recent progress with the help of lattice QCD (see [47,48] for recent reviews), some of the LECs are still poorly known. Furthermore, the effects of chiral logarithms, which carry a scale dependence, are often non-negligible [46, 47. Using the values from one of the fits reported in [47] for the LECs evaluated at the scale of the $\rho$ mass, we obtain the following values for our three relevant combinations:

$$
\begin{aligned}
& a \simeq-0.9 \times 10^{-3}, \\
& b \simeq-0.9 \times 10^{-3}, \\
& c \simeq+1.7 \times 10^{-3},
\end{aligned}
$$

where we have used $m_{\pi}=140 \mathrm{MeV}$ and $f_{\pi}=92 \mathrm{MeV}$.

Using these values, we find that the NLO corrections shift the second-order transition to $\gamma_{c} \simeq 0.99$. However, when rescaling the control parameter we obtain $\gamma_{c}^{R} \simeq 1$.

Let us now explore what happens for different values of the NLO parameters. Since $b$ enters with a minus sign 

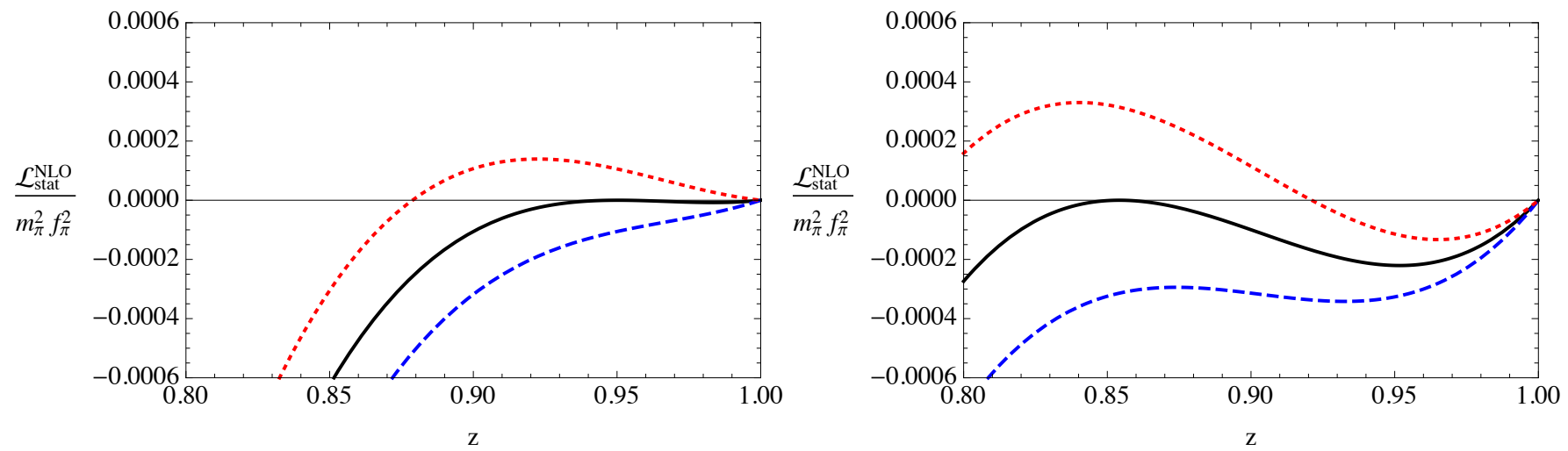

Fig. 2. Lagrangian density, given in Eq. (58), as a function of the variational parameter $z=\cos \alpha$ considering two different values of $t$, see Eq. (63). The different lines correspond to values of $\gamma$ close to the phase transition between the normal phase and the pion condensed phase. To be more specific, the dotted (red) lines correspond to $\gamma=\gamma_{c}+0.001$, the solid (black) lines correspond to $\gamma=\gamma_{c}$ and the dashed (blue) lines have been obtained with $\gamma=\gamma_{c}-0.001$. The maximum of $\mathcal{L}_{\text {stat }}^{\mathrm{NLO}}$ corresponds to the NLO pressure of the system. Left panel: results obtained taking $t=0.018$, the first order phase transition leads to a change $\Delta z \simeq 0.05$ of the tilting angle of the pion condensate. Right panel: results obtained taking $t=0.02$, the first order phase transition corresponds to a change $\Delta z \simeq 0.15$ of the pion condensate.

in Eq. (60) and to simplify the analysis, we limit ourselves to a particular parameter subspace characterized by

$$
t=a=-b=c .
$$

Clearly, a more refined analysis can be done, however, especially considering the uncertainty on the values of the LECs, it seems to us more appropriate to conduct a qualitative study in terms of one single variable.

In Fig. 11we report the value of $\gamma_{c}$ (solid black line) as a function of $t$. The vertical dotted line corresponds to the onset of the first order phase transition: the second order phase transition turns into a first order phase transition for $t \gtrsim 0.017$. We note that depending on the sign of $t$, $\gamma_{c}$ can be larger or smaller than 1 , however $\gamma_{c}^{R} \simeq 1$ for any considered value of $t$. This suggests that the phase transition happens at $\mu_{I}=m_{\pi, 4}$.

In order to better understand the effect of the NLO corrections on the order of the phase transition, we report in Fig. 2 the plot of the static Lagrangian for $t=0.018$, left panel, and for $t=0.02$, right panel. We consider three values of $\gamma$ : below the phase transition point (dashed line), at the phase transition point (solid line) and in the pion condensed phase (dotted line). Close to the phase transition the vacuum and the LO pressures balance. Therefore the quantity reported in Fig. 2 basically corresponds to the NLO contribution, generating a small additional structure inducing a weak first-order transition. The pressure of the system corresponds to the maximum of the static Lagrangian and is obviously a continuous function of $\gamma$. However, the tilting angle $\alpha$ of the ground state, see Eq. (4), is discontinuous. This is accompanied by a discontinuity in the number density with a jump $\Delta n_{I} \propto \sqrt{\Delta z}$. Present lattice QCD simulations, see [5, use $m_{\pi}=390$ $\mathrm{MeV}$, but do not seem to show any jump in $n_{I}$ at the phase transition point. It might be of interest to implement lattice QCD simulations with a larger value of the pion mass. In this case the physical value of the phase transition point does not change much because $\gamma_{c}^{R} \simeq 1$, however the phase transition might turn to be of the first order type, because a larger pion mass implies a larger $t$.

\section{Conclusions}

The properties of matter at nonvanishing isospin chemical potential are quite interesting and in some aspects highly non-trivial. We have shown that the formation of the pion condensate can be described by a standard GP Lagrangian, in which all the coefficients depend on $\mu_{I}$. This is one of the reasons why the description of the condensation mechanism looks complicated. The GP approximation breaks down deep in the pion condensed phase, because it corresponds to a low-density approximation and the number density of pions in the ground state grows with $\mu_{I}$. For this reason we have obtained a different expansion of the $\chi \mathrm{PT}$ Lagrangian, in which the identification of the massless NGB is more direct than in the standard approach. This new approach leads to a Lagrangian similar to the one used for describing quantum magnets, with the isospin playing the role of the spin in condensed matter systems. The broken phase can be identified with an ordered magnetic phase in which the isospins are aligned along the direction of the isospin chemical potential. Indeed, the isospin chemical potential enters in the chiral Lagrangian as an external source, pretty much as the magnetic field enters in the Lagrangian of a quantum magnet. A positive chemical potential leads to the alignment of $\pi_{+}$ mesons, that corresponds to a $\left\langle\pi_{+}\right\rangle$condensate. On the other hand, a negative chemical potential aligns the $\pi_{-}$ mesons, corresponding to a $\left\langle\pi_{-}\right\rangle$condensate. We have obtained all the interaction and surface terms of the soft Lagrangian by integrating out the radial fluctuations around the vev. The kinetic and interaction terms can be written in the compact form reported in Eq. (50), in which we 
made use of the acoustic metric emerging from the interaction of the NGB with the vacuum fluctuations.

Finally, we have tested the order of the phase transition between the normal phase and the pion condensed phase including NLO chiral corrections. For standard values of the LECs the transition remains of the second order, but an intriguing possibility is that lattice QCD simulations with a very large pion mass might observe a first order phase transition. Moreover, more refined lattice QCD simulations could be used to determine the combinations of the LECs that appear in the NLO chiral Lagrangian.

\section{Appendix A: Self-bound pion stars}

Since in the $\pi c$ phase one of the charged pions is stable [27, we speculate on the possible existence of stars consisting of condensed pions. We will assume a weak first order phase transition between the normal phase and the $\pi c$ phase, meaning that we focus on the region $t \gtrsim 0.017$, see Sec. 5 probably corresponding to unphysical values for the LECs.

This system, if sufficiently cold, will be self-bound and it would not spread as a gas even if it is so small to be gravitationally unbound. The resulting stellar object would be a particular type of Bose star. Bose stars are stellar objects consisting of a large number of bosons in which the boson wave-function varies inside the star, see for example [49]. For noninteracting bosons with mass $m$, the maximum mass of a Bose star has been determined in [50] and turns out to be $M_{\max }=0.633 /(G m)$, where $G$ is the gravitational constant. For self-interacting bosons, the maximum mass can be larger, as shown in [51] for axion stars. Certainly, Bose stars can only exist if a stable boson exists.

Let us assume that matter in the $\pi \mathrm{c}$ phase is produced by some astrophysical event. Since it is self-bound it will not evaporate and can possibly accrete matter capturing positrons, converting them in $\pi_{+}$and ejecting neutrinos. In other words, a small number of pions produced with a certain asymmetric mechanism in such a way that there are, say, much more $\pi_{+}$than $\pi_{-}$(meaning that $\mu_{I}>0$ ) may become larger and larger if the $\pi_{+}$condense. Indeed, if this chunk of matter is sufficiently cold, the $\pi_{+}$will form a condensate and will not decay in leptons [27. On the other hand the $\pi_{-}$do not condense and will quickly decay, mainly in muons and corresponding neutrinos [27. However, we expect that the system does not eject electrically charged particles because the electrons produced by the muon decay will be bound by the electromagnetic force.

The neutrality condition for this peculiar system reads

$$
n_{I}=n_{e}
$$

where $n_{e}$ is the electron number density. Although the isospin chemical potential and the electron chemical potential are not equal, a relation between them can be obtained once the number densities are expressed in terms of the corresponding chemical potentials. At $T=0$ the process $e^{-}+\pi_{+} \rightarrow \nu_{e}$ cannot happen because all pions are in the condensate, corresponding to a state of zero momentum. However, at $T>0$, part of the $\pi_{+}$are in excited states and can be annihilated by electrons in neutrinos. These neutrinos will certainly escape leading to a cooling of the stellar object and a related reduction of the excited $\pi_{+}$. For this reason we can conveniently consider the $T=0$ case, meaning that no quasiparticle thermal excitation is present.

If the system is sufficiently big it can certainly gravitationally capture neutral particles, like neutrons or atoms, but in the following we will assume the simplified scenario that it only consists of $e^{-}$and condensed $\pi_{+}$.

Solving the Tolman-Oppenheimer-Volkoff equation, see for example [52, one can determine the mass-radius sequence for a system of pions in the $\pi \mathrm{c}$ phase neutralized by a gas of electrons. Since matter is self-bound we expect a mass-radius trajectory similar to that of strangestars [53, see for example [54]. The maximum mass of these stellar objects depends on the various parameters of the pion pressure. As a preliminary result we have found that using $m_{\pi}=140 \mathrm{MeV}, f_{\pi}=92 \mathrm{MeV}$ and $t=0.02$, stellar masses up to few times the solar mass with radii of tens of kilometers can be formed. Further analysis is ongoing and we expect to report on this topic in the future.

\section{Appendix B: More about the alternative de- scription of the $\pi c$ phase}

Let us clarify some aspects of the procedures presented in Sec. 4 that might be of some concern.

First of all, in the expansion of the potential in Eq. (42) we have neglected terms of order $\chi^{3}$ and $\chi^{2} \partial_{0} \hat{\varphi}_{2}$. To make sure that our approach is correct, we derive from Eq. (43) the equation of motion of the $\chi$ field,

$$
\left(\square+m_{\pi}^{2} \frac{\gamma^{4}-1}{\gamma^{2}}\right) \chi=\mu_{I} \sin (2 \bar{\rho})\left(\hat{\varphi}_{1} \partial_{0} \hat{\varphi}_{2}-\hat{\varphi}_{2} \partial_{0} \hat{\varphi}_{1}\right),
$$

that we can rewrite using Eq. (35) as

$$
\left(\square+m_{\pi}^{2} \frac{\gamma^{4}-1}{\gamma^{2}}\right) \chi=\mu_{I} \sin (2 \bar{\rho}) \partial_{0} \arcsin \left(\hat{\varphi}_{2}\right),
$$

meaning that for any $\gamma>1$ and at the leading derivative order, $\chi \propto \partial_{0} \hat{\varphi}_{2}$. Therefore both $\chi^{3}$ and $\chi^{2} \partial_{0} \hat{\varphi}_{2}$ terms are $\mathcal{O}\left(p^{3}\right)$ and should not be included in the $\mathcal{O}\left(p^{2}\right)$ Lagrangian.

The second aspect that deserves some comment is related to the fate of the $\pi_{0}$ field. In Sec. 4 we have neglected this mode, but it can be straightforwardly included by considering $\hat{\varphi}=\left(\hat{\varphi}_{1}, \hat{\varphi}_{2}, \hat{\varphi}_{3}\right)$. This mode does not couple with the radial fluctuations, because of the Levi-Civita symbol in Eq. (45). Therefore, it does not feel the background fluctuations leading to the renormalization of the propagation speed. In other words, the Lagrangian of this field is simply given by a standard Lorentz invariant expression. In writing the Lagrangian including this mode it is 
important to consider that the potential term in Eq. 37 must be written in the appropriate way

$$
V=-f_{\pi}^{2} m_{\pi}^{2}\left(\cos \rho+\left(\hat{\varphi}_{1}^{2}+\hat{\varphi}_{2}^{2}\right) \frac{\gamma^{2}}{2} \sin ^{2} \rho\right)
$$

because now $\hat{\varphi}_{1}^{2}+\hat{\varphi}_{2}^{2} \neq 1$. In both the normal phase and the broken phase we can write the quadratic Lagrangian in the separable form

$$
\mathcal{L}=\mathcal{L}_{\varphi_{1}, \varphi_{2}}+\mathcal{L}_{\varphi_{3}}
$$

thus we can restrict, at the quadratic order, to considering the $\mathcal{L}_{\varphi_{1}, \varphi_{2}}$ Lagrangian. At higher orders in the fields this separation is no longer possible. However, in the broken phase $m_{\pi^{0}}=\mu_{I}$, therefore, this mode decouples from the soft-Lagrangian when considering momenta and energies below this scale. For this reason the soft Lagrangian in Eq. (50) is valid for momenta much smaller than $\mu_{I}$.

Finally, let us sketch how the standard expression of the quadratic Lagrangian, as given for example in 27, can be obtained starting from the expression in terms of radial and angular fields. We focus on the normal phase (in the broken phase a similar reasoning can be used). The leading order Lagrangian is given by

$\mathcal{L}=\frac{f_{\pi}^{2}}{2}\left(\partial^{\mu} \chi \partial_{\mu} \chi+\chi^{2} \partial^{\mu} \hat{\varphi}_{i} \partial_{\mu} \hat{\varphi}_{i}-2 m_{\pi} \gamma \chi^{2} \epsilon_{3 i j} \hat{\varphi}_{i} \partial_{0} \hat{\varphi}_{j}\right)-V(\chi)$

with the potential term in Eq. (67). By definition $\varphi_{i}=$ $\chi \hat{\varphi}_{i}$, then considering that

$$
\partial^{\mu} \chi \partial_{\mu} \chi+\chi^{2} \partial^{\mu} \hat{\varphi}_{i} \partial_{\mu} \hat{\varphi}_{i}=\partial^{\mu} \varphi_{i} \partial_{\mu} \varphi_{i}
$$

we obtain the quadratic Lagrangian

$$
\begin{aligned}
\mathcal{L} & =\frac{f_{\pi}^{2}}{2}\left(\partial^{\mu} \varphi_{i} \partial_{\mu} \varphi_{i}-2 m_{\pi} \gamma \epsilon_{3 i j} \varphi_{i} \partial_{0} \varphi_{j}\right) \\
& +f_{\pi}^{2} m_{\pi}^{2}\left(1-\frac{\varphi_{3}^{2}}{2}-\frac{1-\gamma^{2}}{2}\left(\varphi_{1}^{2}+\varphi_{2}^{2}\right)\right)
\end{aligned}
$$

that is the standard expression for the $O\left(p^{2}\right)$ quadratic Lagrangian. Note that in the normal phase one can distinguish three modes, but only one mode appears in the expression of the Lagrangian in Eq. (50). It seems, therefore, that two modes disappear in the transition from the normal phase to the broken phase. As discussed above, one of this mode is related to the $\pi_{0}$, that decouples. The second mode that disappears is actually integrated out. Indeed, at the phase transition point the radial mode develops a nontrivial vev and, as shown in Sec. 4, the small fluctuations around it can be integrated out. Note that the mode that is integrated out is not one of the two charged modes, instead it is given by the combination of the charged modes that corresponds to the radial fluctuation.

\section{References}

1. D. Son, M.A. Stephanov, Phys.Rev.Lett. 86, 592 (2001), arXiv: hep-ph/0005225
2. J. Kogut, D. Toublan, Phys.Rev. D64, 034007 (2001), arXiv: hep-ph/0103271

3. M.G. Alford, A. Kapustin, F. Wilczek, Phys.Rev. D59, 054502 (1999), arXiv:hep-lat/9807039

4. J. Kogut, D. Sinclair, Phys.Rev. D66, 034505 (2002), arXiv:hep-lat/0202028

5. W. Detmold, K. Orginos, Z. Shi, Phys. Rev. D86, 054507 (2012), arXiv: 1205.4224

6. W. Detmold, K. Orginos, M.J. Savage, A. Walker-Loud, Phys. Rev. D78, 054514 (2008), arXiv:0807.1856

7. P. Cea, L. Cosmai, M. D'Elia, A. Papa, F. Sanfilippo, Phys. Rev. D85, 094512 (2012), arXiv: 1202.5700

8. G. Endrödi, Phys. Rev. D90(9), 094501 (2014), arXiv: 1407.1216

9. D. Toublan, J. Kogut, Phys.Lett. B564, 212 (2003), arXiv:hep-ph/0301183

10. A. Barducci, R. Casalbuoni, G. Pettini, L. Ravagli, Phys.Rev. D69, 096004 (2004), arXiv:hep-ph/0402104

11. A. Barducci, R. Casalbuoni, G. Pettini, L. Ravagli, Phys.Rev. D71, 016011 (2005), arXiv:hep-ph/0410250

12. D. Ebert, K.G. Klimenko, Eur. Phys. J. C46, 771 (2006), arXiv: hep-ph/0510222

13. D. Ebert, K.G. Klimenko, J. Phys. G32, 599 (2006), arXiv:hep-ph/0507007

14. L.y. He, M. Jin, P.f. Zhuang, Phys.Rev. D71, 116001 (2005), arXiv:hep-ph/0503272

15. L. He, Phys. Rev. D82, 096003 (2010), arXiv:1007.1920

16. B. Klein, D. Toublan, J. Verbaarschot, Phys.Rev. D72, 015007 (2005), arXiv:hep-ph/0405180

17. T. Kanazawa, T. Wettig, JHEP 10, 55 (2014), arXiv: 1406.6131

18. T. Graf, J. Schaffner-Bielich, E.S. Fraga, Phys. Rev. D93(8), 085030 (2016), arXiv:1511.09457

19. M. Loewe, C. Villavicencio, Phys.Rev. D67, 074034 (2003), arXiv:hep-ph/0212275

20. M. Loewe, C. Villavicencio, Phys.Rev. D70, 074005 (2004), arXiv:hep-ph/0404232

21. T. Xia, P. Zhuang (2014), arXiv:1411.6713

22. M. Loewe, A. Raya, C. Villavicencio (2016), arXiv: 1610.05751

23. D. Ebert, N.V. Gubina, K.G. Klimenko, S.G. Kurbanov, V.C. Zhukovsky, Phys. Rev. D84, 025004 (2011), arXiv: 1102.4079

24. S. Carignano, E.J. Ferrer, V. de la Incera, L. Paulucci, Phys. Rev. D92(10), 105018 (2015), arXiv: 1505. 05094

25. D. Nowakowski, M. Buballa, S. Carignano, J. Wambach, in Proceedings, Compact Stars in the QCD Phase Diagram IV (CSQC (2015), arXiv: 1506.04260

26. P. Adhikari, J.O. Andersen (2016), arXiv:1610.01647

27. A. Mammarella, M. Mannarelli, Phys. Rev. D92(8), 085025 (2015), arXiv: 1507.02934

28. J. Gasser, H. Leutwyler, Annals Phys. 158, 142 (1984)

29. H. Leutwyler, Annals Phys. 235, 165 (1994), arXiv:hep-ph/9311274

30. G. Ecker, Prog.Part.Nucl.Phys. 35, 1 (1995), arXiv: hep-ph/9501357

31. S. Scherer, Adv.Nucl.Phys. 27, 277 (2003), arXiv: hep-ph/0210398

32. S. Carignano, A. Mammarella, M. Mannarelli, Phys. Rev. D93(5), 051503 (2016), arXiv: 1602.01317

33. J.O. Andersen, Rev. Mod. Phys. 76, 599 (2004), arXiv: cond-mat/0305138

34. J.S.R. Chisholm, Nucl. Phys. 26(3), 469 (1961) 
35. S. Kamefuchi, L. O'Raifeartaigh, A. Salam, Nucl. Phys. 28, 529 (1961)

36. S. Weinberg, Physica A96, 327 (1979)

37. T. Schäfer, D.T. Son, M.A. Stephanov, D. Toublan, J.J.M. Verbaarschot, Phys. Lett. B522, 67 (2001), arXiv:hep-ph/0108210

38. F. Dalfovo, S. Giorgini, L.P. Pitaevskii, S. Stringari, Rev. Mod. Phys. 71, 463 (1999)

39. F.D.M. Haldane, Phys. Rev. Lett. 50, 1153 (1983)

40. F.D.M. Haldane, Physics Letters A 93(9), 464 (1983)

41. I. Affleck, Nucl. Phys. B257, 397 (1985)

42. F.D.M. Haldane, J. Appl. Phys. 57, 3359 (1985)

43. I. Affleck, Les Houches 1988, Session 49, 563 (1988)

44. M. Mannarelli, C. Manuel, Phys. Rev. D77, 103014 (2008), arXiv: 0802.0321

45. C. Barcelo, S. Liberati, M. Visser, Living Rev. Rel. 8, 12 (2005), [Living Rev. Rel.14,3(2011)], arXiv: gr-qc/0505065

46. J. Gasser, H. Leutwyler, Nucl. Phys. B250, 465 (1985)

47. J. Bijnens, G. Ecker, Ann. Rev. Nucl. Part. Sci. 64, 149 (2014), arXiv: 1405.6488

48. S. Aoki et al., Eur. Phys. J. C74, 2890 (2014), arXiv: 1310.8555

49. T.D. Lee, Y. Pang, Phys. Rept. 221, 251 (1992)

50. J.D. Breit, S. Gupta, A. Zaks, Phys. Lett. B140, 329 (1984)

51. E. Braaten, A. Mohapatra, H. Zhang, Phys. Rev. Lett. 117, 121801 (2016), arXiv:1512.00108

52. S.L. Shapiro, S.A. Teukolsky, Black holes, white dwarfs, and neutron stars: The physics of compact objects (1983)

53. C. Alcock, E. Farhi, A. Olinto, Astrophys.J. 310, 261 (1986)

54. M. Mannarelli, G. Pagliaroli, A. Parisi, L. Pilo, Phys.Rev. D89, 103014 (2014), arXiv:1403.0128 